\title{
An Independent View?
}

\author{
David L. Lambert
}

Department of Astronomy, University of Texas, Austin, TX 78712-1083

\begin{abstract}
The current dispute over the oxygen abundance in metalpoor stars has been viewed from a distance by this observer who would claim a measure of independence despite an intermittent interest in oxgen abundance determinations over 3 decades. This summary attempts to persuade all participants whether they advocate a 'high' or 'low' oxygen abundance or are simply bemused by the dispute that in resolving the present disagreements we shall learn not only about the oxygen abundance of these oldest Galactic stars but shall achieve a deeper understanding of the atmospheres of the stars.
\end{abstract}

\section{Introduction}

In this auditorium which is licensed 'pursuant to act of parliament for public music and dancing', we have been entertained by arguments for and against a 'high' oxygen abundance in metal-poor stars, assured that the question of the true oxygen abundance is vital to understanding the ages of stars, and exposed to the implications for Galactic chemical evolution and nucleosynthesis by Type II supernovae. As a person who earns a living from abundances analyses of stars, the principal immediate lesson to be drawn from today's 'music and dancing' would seem to be that not a single participant switched parties in the parliamentary sense. The oxygen problem remains unresolved.

Yet, is it not remarkable that today, when all the ingredients used in quantitative stellar spectroscopy of very metal-poor stars are so refined, that the abundance of oxygen, a common and astrophysically crucial element, remains uncertain by a large factor with different authors using different spectroscopic signatures insisting that their abundance determinations are subject to uncertainties much less than the large factor between alternative analyses? This question leads to supplementary questions. For example, is the root cause of the problem peculiar to oxygen? Or are there deficiencies in our analytical tools that should cause us to ponder the accuracy of all abundance estimates?

Embedded as we may be currently in the debate over the oxygen abundance, we should not forget that there have been other debates as heated and as puzzling to the disputants but which were resolved and are now no more than a speck in the history of stellar spectroscopy. In this context, I recall three. Goldberg, Müller, and Aller's (1960) classic analysis of the solar composition yielded a $\mathrm{Fe} / \mathrm{Si}$ ratio that was about one-tenth the meteoritic ratio. The 'low' photospheric $\mathrm{Fe}$ abundance generated a heated debate that was cooled considerably when it was realised that GMA's adopted $g f$-values were in error. A 
discrepancy over the metallicity of the Hyades cluster derived from photometry and spectroscopy simmered in the literature in the 1970s but is now largely forgotten as a concensus was reached. The question of super-metal-rich (SMR) stars was aired also in the 1970 s literature. Now, while few doubt that stars with $[\mathrm{Fe} / \mathrm{H}] \sim 0.4$ exist, arguments may continue about whether a particular star is or is not a SMR star. Perhaps, a key contributor to the uncertainty was the fact that the spectrum of a SMR cool giant - $\mu$ Leonis, for example - is so cluttered with weak $\mathrm{CN}$ and other lines that, unless the spectrum is of high-resolution and high $\mathrm{S} / \mathrm{N}$ ratio, the continuum may be drawn too low and the equivalent width of lines underestimated.

As these debates passed into history so will the oxygen problem. If the resolution is rooted in subtleties associated with oxygen atoms or molecules, the oxygen problem will likely be relegated to a footnote, but, if resolution of the problem is to be found in the employment of classical model atmospheres, the oxygen problem should be featured in the main body of the text describing the growth of quantitative stellar spectroscopy.

\section{Basic Ingredients}

In the extraction of an oxygen abundance from a stellar spectrum, I would identify three necessary ingredients (and sources of uncertainty!): the spectrum that is the source of the line identified with oxygen; the basic atomic or molecular data pertinent to the line and its carrier; the model atmosphere with which the line strength and basic data are combined to obtain the abundance, here the $\mathrm{O} / \mathrm{H}$ ratio.

Although I shall not review in detail each of the indicators of the oxygen abundance, a list will be helpful. In order of increasing wavelength, the principal lines are: the $\mathrm{OH} \mathrm{A}^{2} \Sigma^{+}-\mathrm{X}^{2} \Pi \Delta v=0$ lines near $3100 \AA$, the [O I] 6300 and $6363 \AA$ lines, the $\mathrm{O}_{\mathrm{I}} 7770 \AA$ lines, and the $\mathrm{OH}$ vibration-rotation overtone lines of the $\mathrm{X}^{2} \Pi$ state near $1.6 \mu \mathrm{m}$. For each set of lines, the identifications are secure, and the measurements of equivalent widths are reliable. Adverse comments have appeared concerning the $\mathrm{OH}$ ultraviolet lines but, in the case of the extremely metal-poor stars where the oxygen problem is the most acute, there is an adequate set of well resolved $\mathrm{OH}$ lines and the continuum can be defined with good precision. The observational fact that the [O I] lines are detectable in very metal-poor giants but are too weak for detection in very metal-poor dwarfs has featured in the debate (see below); identification of the [O I] lines has not been questioned, however. In short, I do not think that the origins of the oxygen problem are to be found at this early stage of the abundance analysis.

Basic atomic/molecular data for the [O I], O I, and $\mathrm{OH}$ transitions are known reliably from either laboratory and/or quantum calculations. While some diatomic molecules of astrophysical interest lack basic data of the desired accuracy (e.g., do we really know the dissociation energy of CN and the $f$-value of CN Red system to the accuracy needed to get $\mathrm{N}$ abundances for cool giants?), the data for $\mathrm{OH}$ is secure. It is true that published analyses of the $\mathrm{OH}$ ultraviolet lines rely (surprisingly!) on determinations of the $g f$-values from fits to the solar spectrum, the resultant values are (surprisingly!) not very different from laboratory/theoretical values. 
By elimination, I surmise that the root cause of the oxygen problem is to be found among the many constituents comprising that ingredient I listed simply as 'model atmosphere'. Published oxygen abundances have all been based on classical atmospheres, i.e., atmospheres built from the assumptions of planeparallel homogeneous layers in hydrostatic, local thermodynamic, and radiative/convective equilibrium. Indeed, the models used have been either from the Kurucz or Uppsala grids. With few exceptions, these models have been combined with a line analysis code embodying also local thermodynamic equilibrium. The oxygen problem is not traceable to differences in classical model atmospheres or line analysis programs.

In such a 'standard' scheme of abundance analysis, there are numerous sources of uncertainty: notably, the choice of the defining parameters $\left(T_{\text {eff }}, \log g\right.$, composition, microturbulence), and the continuous opacity (a potential problem only for the $\mathrm{OH}$ ultraviolet lines). Amelioration of the oxygen problem is certainly possible through particular choices of defining parameters: Gustafsson's contribution was along these lines, and one $\mathrm{OH}$ advocate very briefly showed how King (2000) had been able to reduce the oxygen abundance based on the $\mathrm{OH}$ ultraviolet lines to the lower values indicated by $\left[\mathrm{O}_{\mathrm{I}}\right]$ lines.

Some analyses of the $\mathrm{O}_{\mathrm{I}}$ lines have considered line formation under nonLTE conditions in standard model atmospheres (Kiselman, these proceedings) but this does not resolve the oxygen problem, not only are the non-LTE effects on these lines small but the $\mathrm{OH}$ lines (not yet examined for non-LTE effects) are a major part of the problem, and the [O I] lines, the other major part, are surely immune to non-LTE effects. Although the $\mathrm{OH}$ ultraviolet lines are candidates for substantial non-LTE effects, the $\mathrm{OH}$ infrared lines are not (Hinkle \& Lambert 1975). Measurements reported here on the $\mathrm{OH}$ infrared lines suggest that they provide an oxygen abundance similar to but slightly less than that from the $\mathrm{OH}$ ultraviolet lines (Balachandran; Melendez \& Barbuy); the severity of the oxygen problem is reduced somewhat but not eliminated if the $\mathrm{OH}$ infrared lines are preferred over the $\mathrm{OH}$ ultraviolet lines.

This chain of reasoning leads to a suspicion that the classical atmospheres are at the root of the problem. Before discussing supporting theoretical evidence presented eloquently here by Asplund, I shall look at the question of oxygen abundance determinations from the perspective of an independent observer wishing to obtain results that are minimally sensitive to the secret goings-on in a stellar atmosphere.

\section{The Case for the $\left[\mathrm{O}_{\mathrm{I}}\right]$ Lines}

Access to computer packages that are all too commonly treated as black boxes of impeccable reliability and applicability seems to freeze the mind. There appears to be a reluctance to make 'back-of-the-envelope' assessments of potential uncertainties. In the latter spirit, I offer the following statements about the stars that form the basis for the oxygen problem. My remarks were anticipated largely by our President in his opening remarks (Kraft, these proceedings):

- The [O I] Lines. Oxygen predominantly resides as neutral atoms in the atmosphere of a metal-poor dwarf or giant; $\mathrm{CO}$ molecules soak up few oxygen atoms. The neutral atoms are overwhelmingly in the ground $\left(2 p^{4}\right)$ 
configuration. Levels of the ground configuration being connected by forbidden transitions are in LTE. The strength of an [O I] line is insensitive to the atmospheric temperature profile but is sensitive to the pressure profile.

- The OH Ultraviolet and Infrared Lines. OH molecules in the stars observed todate are a trace species. Molecule formation is very sensitive to the atmospheric temperature and pressure profiles. Non-LTE effects are likely for the $\mathrm{OH}$ ultraviolet lines.

- The $\mathbf{O}_{\text {I }}$ Lines. These lines contributed by the very few oxygen atoms in highly-excited levels are dependent on the structure of the deeper layers of the atmosphere, and potentially sensitive to non-LTE effects.

The oxygen problem is most commonly stated in terms of the trend of the $\mathrm{O} / \mathrm{Fe}$ ratio with $[\mathrm{Fe} / \mathrm{H}]$. Stated as $[\mathrm{O} / \mathrm{Fe}]$, one aspect of the problem is that the OH ultraviolet analyses (Israelian et al. 1998; Boesgaard et al. 1999; also these proceedings) show $[\mathrm{O} / \mathrm{Fe}]$ to increase linearly with decreasing $[\mathrm{Fe} / \mathrm{H}]$ with a slope of about 0.4 in contrast to the alternative view based originally on [O I] measurements in giants (Barbuy 1988) that $[\mathrm{O} / \mathrm{Fe}]$ at about 0.4 is approximately independent of $[\mathrm{Fe} / \mathrm{H}]$ for $[\mathrm{Fe} / \mathrm{H}] \leq-1$ : the difference in $[\mathrm{O} / \mathrm{Fe}]$ at the limit to which the $\mathrm{OH}$ observations have been pushed $([\mathrm{Fe} / \mathrm{H}] \simeq-3)$ is about $0.7 \mathrm{dex}$ or a factor of 5 . For $[\mathrm{Fe} / \mathrm{H}] \geq-1$, the $\mathrm{OH}$ ultraviolet lines give an abundance consistent with that from $\left[\mathrm{O}_{\mathrm{I}}\right]$ lines from dwarf and giant stars: $[\mathrm{O} / \mathrm{Fe}]$ declines smoothly from about 0.4 to zero at $[\mathrm{Fe} / \mathrm{H}]=0.0$.

If $[\mathrm{O} / \mathrm{Fe}]$ is considered, the pressure sensitivity of the $[\mathrm{OI}]$ lines is surely reduced, if not eliminated, by considering these lines along with $\mathrm{Fe}$ II lines of a similar wavelength. This consideration also reduces the effects of non-LTE as they affect iron atoms and ions. Empirical and theoretical evidence suggests that non-LTE effects on Fe I lines in metal-poor stars may be substantial but of only minor consequence for Fe II lines (e.g., Thévenin \& Idiart 1999). In short, if the weak [O I] lines are measureable with confidence in spectra of metal-poor stars, their analysis along with Fe II lines is expected to provide an unimpeachable result for $[\mathrm{O} / \mathrm{Fe}]$ versus $[\mathrm{Fe} / \mathrm{H}]$. A corollary follows: if other lines give different results for $[\mathrm{O} / \mathrm{Fe}]$, the likely explanation is that inappropriate atmospheric structures were chosen. Reasons behind the choice of atmosphere include: use of Fe I lines in the LTE analysis to get the Fe abundance or to set the atmospheric defining parameters, adoption of classical atmospheres with incorrect defining parameters, failure of the true atmosphere to conform to a classical atmosphere, etc.

The qualitative ('back-of-the-envelope') arguments in favor of the $[\mathrm{O} / \mathrm{Fe}]$ ratios from the combination of $[\mathrm{O} \mathrm{I}]$ and $\mathrm{Fe}$ II lines are supported by calculations using classical model atmospheres. Why, the spectator may ask, are the $[\mathrm{O} / \mathrm{Fe}]$ results from the $\mathrm{O}$ I and $\mathrm{OH}$ lines preferred by some? My reading of the papers suggests these common adverse criticisms about the use of $\left[\mathrm{O}_{\mathrm{I}}\right]$ lines in very metal-poor stars: (1) the lines are too weak to measure reliably in the most metal-poor star, especially in dwarfs, where the $\mathrm{OH}$ lines are readily measureable, and (2) while the [O I] lines are measureable in giants to low metallicity (say, $[\mathrm{Fe} / \mathrm{H}] \sim-2.5$ ), the line may be filled in by emission or oxygen may have been depleted internally and no longer represents the star's initial abundance. 
Certainly, the [O I] lines are very weak lines in metal-poor dwarf and subgiant stars. Even in the solar spectrum, the $6300 \AA$ line is just $4 \mathrm{~m} \AA$ strong. At this meeting, Nissen, Primas and Asplund showed that a large telescope equipped with a high dispersion spectrograph can push detections of the $6300 \AA$ $\left[\mathrm{O}_{\mathrm{I}}\right]$ in dwarfs down to $[\mathrm{Fe} / \mathrm{H}] \simeq-1.6$ with the precision necessary to discriminate between a high and a low $[\mathrm{O} / \mathrm{Fe}]$ ratio. This set of $[\mathrm{O} / \mathrm{Fe}]$ ratios based on the $[\mathrm{OI}]$ and Fe II lines in dwarfs clearly suggests that the low $[\mathrm{O} / \mathrm{Fe}](\simeq 0.4)$ ratio is correct. It is becoming difficult for an advocate of a high $[\mathrm{O} / \mathrm{Fe}]$ ratio to argue that observations of the [O I] lines in metal-poor dwarfs cannot be made with the precision necessary to distinguish between high and low oxygen abundances. Continued pursuit of the lines in dwarfs may push their detections to $[\mathrm{Fe} / \mathrm{H}] \sim-2$.

In metal-poor giants and subgiants, the low $[\mathrm{O} / \mathrm{Fe}]$ ratio was demonstrated by Barbuy (1988). I can well recall an observing run in which we used the McDonald coudé scanner to measure the $6300 \AA$ [O I] line in the prototypical metal-poor $([\mathrm{Fe} / \mathrm{H}] \simeq-2.7)$ giant HD 122563 (Lambert, Sneden \& Ries 1974). At this meeting, Sneden argued for the low $[\mathrm{O} / \mathrm{Fe}]$ ratio from new observations of the $6300 \AA[\mathrm{OI}]$, the adjacent Sc II, and Fe II lines. As just noted, to nullify this evidence, the arguments are made that either the [O I] absorption lines in giants are prone to filling in by a stellar emission line and/or the surface oxygen abundance has been reduced from a higher initial abundance by nuclear processing and mixing. No concrete evidence has been presented for the emission hypothesis; if emission is weakening an absorption line, is it not odd that the [O I] lines are never seen in emission, and the derived $[\mathrm{O} / \mathrm{Fe}]$ ratios are the same low value to within the measurement errors over the entire sample which spans the metallicity range $-2.5<[\mathrm{Fe} / \mathrm{H}]<-1.0$ ? Reduction of surface oxygen abundance would imply a substantial increase in the surface nitrogen abundance because ON-cycling must be deemed the likely mechanism, and the assumed initial high oxygen abundance greatly exceeds the initial nitrogen abundance. There is no evidence for this increase but, perhaps, the prudent would delay a verdict pending detailed analyses for the $\mathrm{C}, \mathrm{N}$, and $\mathrm{O}$ abundances. But it can hardly be imprudent to point out that mixing must take a special form if the high and steep $[\mathrm{O} / \mathrm{Fe}]$ versus $[\mathrm{Fe} / \mathrm{H}]$ trend is to be transformed to the flat $[\mathrm{O} / \mathrm{Fe}]$ versus $[\mathrm{Fe} / \mathrm{H}]$ trend derived from the observations of $[\mathrm{O} \mathrm{I}]$ in giants.

This observer returning to the question of oxygen abundances of metal-poor stars after two decades of distractions finds it hard to ignore and fault the results obtained for $[\mathrm{O} / \mathrm{Fe}]$ versus $[\mathrm{Fe} / \mathrm{H}]$ from the combination of $[\mathrm{O} \mathrm{I}]$ and $\mathrm{Fe}$ II or Sc II lines. It is difficult to dispute the conclusion that $[\mathrm{O} / \mathrm{Fe}] \sim 0.4$ for $[\mathrm{Fe} / \mathrm{H}]<-1$. A shallow, probably upward, trend of $[\mathrm{O} / \mathrm{Fe}]$ as $[\mathrm{Fe} / \mathrm{H}]$ declines is certainly not ruled out but the high $[\mathrm{O} / \mathrm{Fe}]$ from the $\mathrm{OH}$ analyses is difficult to reconcile with intuition and experience.

\section{Concluding Remarks}

Even if my conclusion about the [O I] lines is correct, the alternative results from $\mathrm{OH}$ and $\mathrm{O}$ I lines call for explanation. Observers' curiosity and theoreticians' unquenchable thirst for reliable data on the compositions of the most metal-poor stars will drive the search for answers to our oxygen problem. A major impetus 
for solving the problem is that observers who seek to push $[\mathrm{O} / \mathrm{Fe}]$ measurements to stars more metal-poor (say, $[\mathrm{Fe} / \mathrm{H}]<-3$ ) than those currently analysed will have to use the $\mathrm{OH}$ ultraviolet lines.

In my discusssion of basic ingredients, I suggested that the answer to the controversy was likely to rest in the representation of the stellar atmosphere. Here, Asplund showed that 3-dimensional hydrodynamic models of metal-poor dwarf stars provide temperature inhomogeneities (and velocity fields) throughout the photosphere, and the principal difference between the 3D model and an equivalent classical model atmosphere is a region of lower temperatures at the top of the 3D photosphere. Not surprisingly, his calculations of the [O I], O I, and $\mathrm{OH}$ ultraviolet lines from the 3D models show that, relative to a classical atmosphere, the abundances derived from [O I] and $\mathrm{O}_{\mathrm{I}}$ lines are little affected but the abundance derived from the ultraviolet $\mathrm{OH}$ lines is significantly reduced; the 3D analysis brings the $\mathrm{OH}$ ultraviolet and $[\mathrm{O} \mathrm{I}]$ results into agreement at the low and flat $[\mathrm{O} / \mathrm{Fe}]$ ratio. It should be noted that the convective flows of the $3 \mathrm{D}$ models result in asymmetric and shifted absorption lines. For the metal-poor turn-off star HD 84937 measured and predicted asymmetries of various Fe I and Fe II lines are in good agreement (Allende Prieto et al. 1999a,b). It should be noted too that the 3D models of the Sun match the solar granulation in amazing detail. In a recent test against high-resolution spectra of Procyon, the models reproduce, as they do for the Sun, the line profiles without introducing microturbulence as a 'fudge' factor. Since in these 3D models there are 'no free parameters' (Asplund, private communication) such as microturbulence and macroturbulence, these successful tests are considered significant evidence for the models.

Not all aspects of the oxygen problem are resolved by discarding classical in favor of the 3D models. Among points for further investigation, I mention an explanation for the high abundances from the $\mathrm{O}$ I lines, an assessment of the effects of non-LTE on the $\mathrm{OH}$ ultraviolet lines for 3D and classical models, and a direct determination of the temperatures of the $\mathrm{OH}$-containing layers using lines across the rotational ladders of several vibrational levels. This latter exercise should be possible from ultraviolet high-resolution spectra from STIS on HST. At the highest STIS resolution, it may be possible to check the predicted line asymmetries of the $\mathrm{OH}$ lines. When $[\mathrm{O} / \mathrm{Fe}]$ estimates are compared, attention must be paid to the determinations of the iron abundance, and, in particular, to possible differences arising from the different sensitivities of Fe I and Fe II lines to non-LTE effects.

Time will tell whether the choice of the low $[\mathrm{O} / \mathrm{Fe}]$ versus $[\mathrm{Fe} / \mathrm{H}]$ trend is correct. Whatever the correct result turns out to be, the observational and theoretical activities leading to the definition and then the resolution of the oxygen problem will lead us to a deeper understanding of stellar atmospheres and their spectra. In time, the oxygen problem for metal-poor stars will be resolved and take a place with the solar iron abundance, the Hyades' metallicity, the composition of the SMR stars, and other spectroscopic puzzles that flickered across the screen of history. It remains to be seen if the oxygen problem is recorded as a footnote, or as marking the transition from the adoption of classical to $3 \mathrm{D}$ model atmospheres in the pursuit of quantitative stellar spectroscopy.

Acknowledgments. I thank the organizers of this joint discussion for their invitation to summarize what has been a very stimulating meeting. I thank too 
the U.S. National Science Foundation, and the Robert A. Welch Foundation for their support of my research in stellar spectroscopy and chemical compositions.

\section{References}

Allende Prieto, C. García López, R., Lambert, D.L., Gustafsson, B. 1999a, ApJ, 526,991

Allende Prieto, C., Asplund, M., García López, R., Gustafsson, B., Lambert, D.L. 1999b, in Stellar Structure: Theory and Tests of Convection in Stellar Structure, ed. A. Giménez et al., ASP Conf. Ser., 173, 205

Barbuy, B. 1988, A\&A, 191, 121

Boesgaard, A.M., King, J.R., Deliyannis, C.P., Vogt, S.S. 1999, AJ, 117, 492

Goldberg, L., Müller, E.A., Aller, L. 1960, ApJS, 5, 1

Hinkle, K.H., Lambert, D.L. 1975, MNRAS, 170, 447

Israelian, G., García López, R., Rebolo, R. 1998, ApJ, 507, 805

King, J.R. 2000, AJ, 120, 1056

Lambert, D.L., Sneden, C., Ries, L.M. 1974, ApJ, 188, 97

Thévenin, F. \& Idiart, J.P. 1999, ApJ, 521, 753 